\title{
Engineering Notes
}

\section{Perpetual Dynamic Soaring in Linear Wind Shear}

\author{
Ricardo Bencatel, $*$ Pierre Kabamba, $\_$and Anouck Girard $\$$ \\ University of Michigan, Ann Arbor, Michigan 48109
}

DOI: $10.2514 / 1 . G 000425$

\begin{tabular}{|c|c|c|}
\hline & & Nomenclature \\
\hline$C_{L}, C_{D}, C_{D_{0}}$ & $=$ & $\begin{array}{l}\text { lift coefficient, drag coefficient, and drag } \\
\text { coefficient with zero lift }\end{array}$ \\
\hline $\mathrm{DS}_{i}$ & $=$ & dynamic soaring number sample \\
\hline$E, E_{\min }$ & $=$ & $\begin{array}{l}\text { aircraft total energy and minimum } \\
\text { sustainable total energy, } \mathrm{J}\end{array}$ \\
\hline$g$ & $=$ & gravity acceleration, $\mathrm{m} / \mathrm{s}^{2}$ \\
\hline$h, h_{\min }$ & $=$ & altitude and safety altitude, $\mathrm{m}$ \\
\hline$K$ & $=$ & drag-polar-induced drag gain \\
\hline$k_{1}, k_{2}, k_{3}$ & $=$ & $\begin{array}{l}\text { necessary and sufficient conditions } \\
\text { inequality parameters }\end{array}$ \\
\hline$L, D$ & $=$ & lift and drag forces, $\mathrm{N}$ \\
\hline$L / D,(L / D)_{\max }$ & $=$ & $\begin{array}{l}\text { lift-to-drag ratio and maximum } \\
\text { lift-to-drag ratio }\end{array}$ \\
\hline $\mathrm{MC}_{i}$ & $=$ & $\begin{array}{l}\text { uniform distribution sample for } \\
\text { Monte Carlo sampling }\end{array}$ \\
\hline$m$ & $=$ & mass, $\mathrm{kg}$ \\
\hline$N$ & $=$ & sample size \\
\hline$S$ & $=$ & wing area, $\mathrm{m}^{2}$ \\
\hline$V_{a}, V_{\min }$ & $=$ & airspeed and stall airspeed, m/s \\
\hline $\boldsymbol{w}, W, W_{x, y, z}$ & $=$ & $\begin{array}{l}\text { wind velocity vector, speed, and velocity } \\
\text { components in } x, y \text {, and } z, \mathrm{~m} / \mathrm{s}\end{array}$ \\
\hline$X, X_{i}$ & $=$ & random variable and sample \\
\hline$x, y, z$ & $=$ & position coordinates, $\mathrm{m}$ \\
\hline$\dot{x}, \dot{y}, \dot{z}$ & $=$ & $\begin{array}{l}\text { velocity components in the ground } \\
\text { frame, } \mathrm{m} / \mathrm{s}\end{array}$ \\
\hline$\ddot{x}, \ddot{y}, \ddot{z}$ & $=$ & $\begin{array}{l}\text { acceleration components in the } \\
\text { ground frame, } \mathrm{m} / \mathrm{s}^{2}\end{array}$ \\
\hline$\beta$ & $=$ & experimental verification confidence level \\
\hline$\gamma_{a}$ & $=$ & flight-path angle relative to the air, rad \\
\hline$\partial w_{\mathrm{LWS}} / \partial h$ & $=$ & wind gradient vector, $\mathrm{s}^{-1}$ \\
\hline$\epsilon$ & $=$ & experimental verification margin of error \\
\hline$\mu, \sigma$ & $=$ & $\begin{array}{l}\text { random variable mean and standard } \\
\text { deviation }\end{array}$ \\
\hline$\rho$ & $=$ & fluid (air) density, $\mathrm{kg} / \mathrm{m}^{3}$ \\
\hline$\phi$ & $=$ & bank angle, rad \\
\hline$\psi, \psi_{W}, \psi_{d W}$ & $=$ & $\begin{array}{l}\text { aircraft heading angle, wind direction, } \\
\text { and wind gradient direction, rad }\end{array}$ \\
\hline
\end{tabular}

Received 3 December 2013; revision received 3 April 2014; accepted for publication 14 April 2014; published online 15 July 2014. Copyright (C 2014 by Ricardo Bencatel, Pierre Kabamba, and Anouck Girard. Published by the American Institute of Aeronautics and Astronautics, Inc., with permission. Copies of this paper may be made for personal or internal use, on condition that the copier pay the $\$ 10.00$ per-copy fee to the Copyright Clearance Center, Inc., 222 Rosewood Drive, Danvers, MA 01923; include the code 1533-3884/ 14 and $\$ 10.00$ in correspondence with the CCC.

*Postdoctoral Researcher, Department of Aerospace Engineering, 1320 Beal Avenue; bencatel@umich.edu. Member AIAA.

†Professor, Department of Aerospace Engineering, 1320 Beal Avenue; kabamba@umich.edu.

${ }^{\ddagger}$ Associate Professor, Department of Aerospace Engineering, 1320 Beal Avenue; anouck@umich.edu. Senior Member AIAA.

\section{Introduction}

$\mathbf{T}$ HIS paper analyzes dynamic soaring in linear wind shear. Wind shear is a type of space and time-dependent airflow vector field. Dynamic soaring is a cyclic flight trajectory that enables energy harvesting from the surrounding flow field. The dynamic soaring trajectory is cyclic and not periodic in the sense that, in each cycle, some of the state variables present the same values and trends for the initial and final conditions. Moreover, for the cyclic trajectory to be part of a sustainable flight, the aircraft energy, sampled at the beginning of a cycle, should be nondecreasing.

This work discusses the necessary and sufficient conditions to enable sustainable flight with dynamic soaring. These conditions allow the aircraft to harvest enough energy from the wind shear to compensate for the energy lost due to drag.

This Note focuses on the study of dynamic soaring in wind shear. The system under analysis is defined by a wind shear model with constant vertical velocity gradient and the aircraft equations of motion, which model the aircraft behavior when subject to a wind gradient. The goal of this study is to define the inequalities that characterize the sufficient and necessary conditions for dynamic soaring. The conditions are applicable to a wide variety of unmanned aerial vehicles (UAVs), allowing the determination of the suitability of an aircraft for dynamic soaring under different environmental conditions.

The equations of flight dynamics based on the flight-path angle and the lift are derived in [1]. There are several studies on optimal dynamic soaring trajectories for energy harvesting from wind shear [2-4]. These studies analyze the evolution of several flight trajectory variables, such as the load factor, climb rate, heading, and bank angle, over the dynamic soaring cycle.

From these studies, only $[2,3]$ study the minimum conditions that allow dynamic soaring to maintain perpetual flight. These studies either refer to specific aerodynamic models (i.e., models with fixed aerodynamic parameters, such as the lift-to-drag ratio, minimum drag coefficient, etc.) or just show the variation of the minimum conditions with the aerodynamic parameters in simplified plots. These results allow a qualitative evaluation of how the conditions for dynamic soaring depend on the aerodynamic parameters. However, they do not allow a quantitative evaluation of this relation for arbitrary aircraft models. As an example, these studies suggest that more aerodynamically efficient aircraft require weaker wind gradients. However, from the presented data, it is difficult to quantify the exact minimum wind gradient or the maximum energy gain from a specific wind gradient, for an arbitrary aircraft model, with specific lift-todrag ratio and minimum drag coefficient.

This work derives the necessary and sufficient conditions for sustainable dynamic soaring as an explicit function of several aircraft and environment parameters. Expressing conditions for dynamic soaring feasibility as an equation that is applicable to most fixed-wing aircraft models is very important to evaluate the suitability of any aircraft for dynamic soaring.

\section{Models}

\section{A. Wind Shear}

Definition II.1: The wind vector is the horizontal component of the air velocity vector at a given position and time:

$$
\boldsymbol{w}:=\left[\begin{array}{c}
W_{x}(\mathbf{x}, t) \\
W_{y}(\mathbf{x}, t)
\end{array}\right]=W(\mathbf{x}, t)\left[\begin{array}{c}
\cos \psi_{W}(\mathbf{x}, t) \\
\sin \psi_{W}(\mathbf{x}, t)
\end{array}\right]
$$




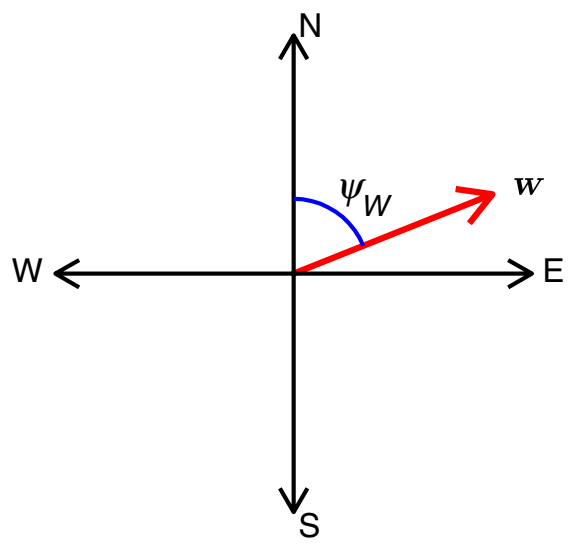

Fig. 1 Wind vector $w$ and direction $\psi_{W}$.

where $W(\mathbf{x}, t)$ is the horizontal wind speed and $\psi_{W}(\mathbf{x}, t)$ is the horizontal wind vector direction relative to the north (Fig. 1).

wind shear is an atmospheric phenomenon that occurs within thin layers separating two regions where the predominant air flows are different, either in speed, in direction, or in both speed and direction. The air layer between these regions usually presents a consistent gradient in the flow field. For simplicity, this study considers only horizontal flow; the wind vector is referred to as $\boldsymbol{w}$ and its total speed as $W$.

Horizontal wind shear is the variation of the horizontal wind with altitude, presenting a vertical wind gradient:

$$
\frac{\partial w(\mathbf{x}, t)}{\partial z} \neq 0
$$

This approach simplifies the phenomenon to uniaxial $z$ wind vector variations and uses a linear layer wind shear model as defined by Sachs and da Costa [4]. The model presents a constant wind gradient, with the wind velocity evolving linearly from the lower to the upper layer:

$$
\boldsymbol{w}(h)=\boldsymbol{w}\left(h_{\min }\right)+\frac{\partial w_{\mathrm{LWS}}}{\partial h}\left(h-h_{\min }\right)
$$

where $\boldsymbol{w}\left(h_{\min }\right)$ is the wind velocity vector at the lower boundary altitude, $h_{\min }$ is the lower boundary of the layer wind shear (LWS),

$$
\frac{\partial \boldsymbol{w}_{\mathrm{LWS}}}{\partial h}=\frac{\mathrm{d} W}{\mathrm{~d} z}\left[\cos \psi_{d W}, \sin \psi_{d W}\right]^{\top}
$$

is a constant wind shear vertical gradient, $[\mathrm{d} W(x, t) / \mathrm{d} z]$ is the vertical wind gradient, and $\psi_{d W}(\mathbf{x}, t)$ is the vertical wind gradient direction (Fig. 2).

\section{B. Aircraft Dynamics}

This work uses an aircraft dynamics model that assumes the presence of an autopilot controlling the low-level dynamics. The velocity equation is

$$
V=\left[\begin{array}{c}
\dot{x} \\
\dot{y} \\
\dot{z}
\end{array}\right]=\left[\begin{array}{c}
\dot{x}_{a} \\
\dot{y}_{a} \\
\dot{z}_{a}
\end{array}\right]+\boldsymbol{w}=V_{a}\left[\begin{array}{c}
\cos \psi \cos \gamma_{a} \\
\sin \psi \cos \gamma_{a} \\
\sin \gamma_{a}
\end{array}\right]+\left[\begin{array}{c}
W_{x} \\
W_{y} \\
W_{z}
\end{array}\right]
$$

where $V=[\dot{x}, \dot{y}, \dot{z}]^{\top}$ is the velocity vector relative to the ground, $\left[\dot{x}_{a}, \dot{y}_{a}, \dot{z}_{a}\right]^{\top}$ is the velocity vector relative to the air (i.e., relative to the flow field), and $\left[W_{x}, W_{y}, W_{z}\right]^{\top}$ is the wind velocity vector. All these vectors are expressed in the ground reference frame, where $x, y$, and $z$ are the north-, east-, and downward directions. $V_{a}$ is a scalar representing the total air relative speed. The air-climb angle $\gamma_{a}$ (Fig. $\underline{3}$ ) is defined as

$$
\gamma_{a}=\arctan \frac{\dot{z}_{V}-W_{z}}{\sqrt{\left(\dot{x}_{V}-W_{x}\right)^{2}+\left(\dot{y}_{V}-W_{y}\right)^{2}}}
$$

Because the autopilot is assumed to control the low-level dynamics, the sideslip is assumed to be regulated and negligible $(\beta \approx 0)$. The equations of motion governing the UAV are (Fig. $\underline{3}$ )

$$
\begin{gathered}
m\left[\begin{array}{c}
\ddot{x} \\
\ddot{y} \\
\ddot{z}
\end{array}\right]=L\left[\begin{array}{c}
-\cos \phi \sin \gamma_{a} \cos \psi-\sin \phi \sin \psi \\
-\cos \phi \sin \gamma_{a} \sin \psi+\sin \phi \cos \psi \\
-\cos \gamma_{a} \cos \phi
\end{array}\right] \\
-D\left[\begin{array}{c}
\cos \gamma_{a} \cos \psi \\
\cos \gamma_{a} \sin \psi \\
-\sin \gamma_{a}
\end{array}\right]+m g\left[\begin{array}{l}
0 \\
0 \\
1
\end{array}\right]
\end{gathered}
$$

where $L$ and $D$ are the aerodynamic lift and drag, $m$ is the aircraft mass, and $g$ is the acceleration of gravity. Differentiating Eq. (4) and combining it with the equations of motion (5), considering $W_{z}=0$ and solving for $\dot{V}_{a}, \dot{\psi}$, and $\dot{\gamma}_{a}$, yields

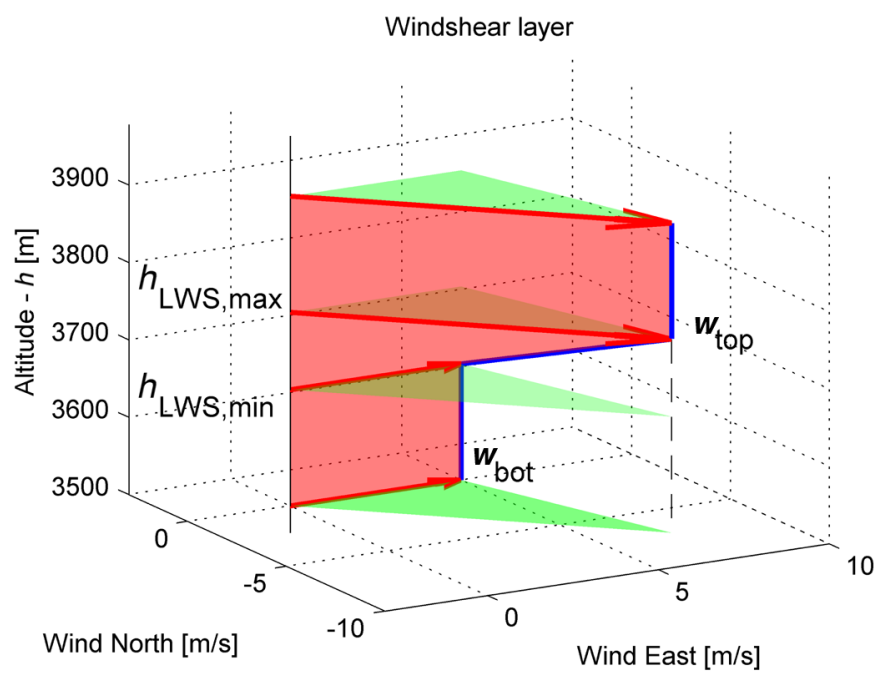

a) Wind vector along the windshear layer

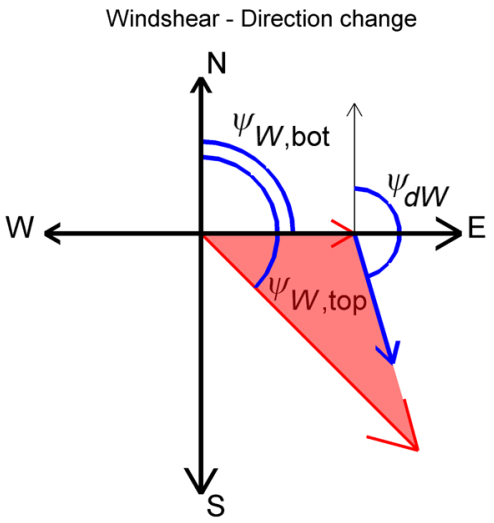

b) Wind direction change along the windshear layer

Fig. 2 Linear layer wind shear model. 


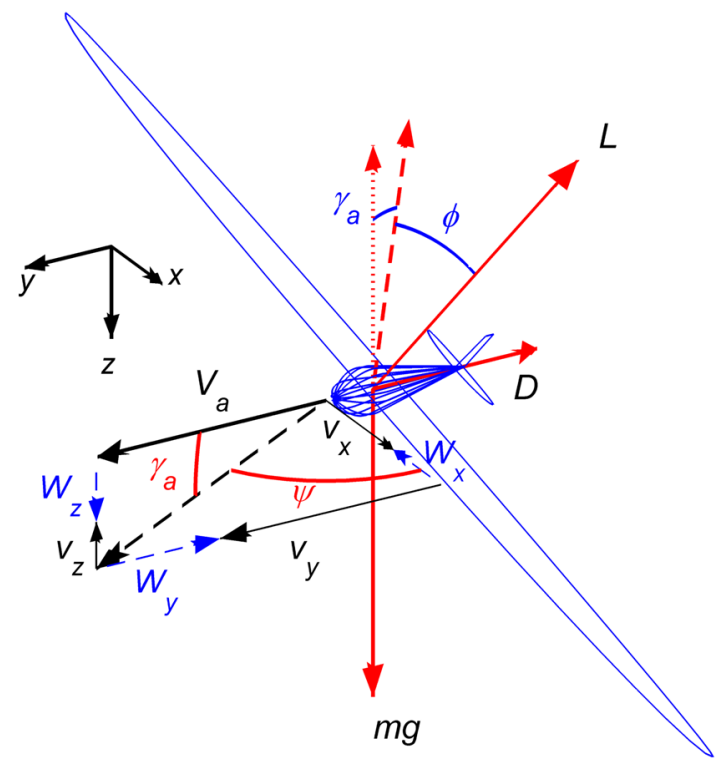

Fig. 3 Wind reference frame and aircraft forces diagram.

$$
\begin{gathered}
\dot{V}_{a}=-\frac{D}{m}-g \sin \gamma_{a}-V_{a} \sin \gamma_{a} \cos \gamma_{a} \cos \left(\psi-\psi_{d W}\right) \frac{\mathrm{d} W}{\mathrm{~d} z} \\
\dot{\gamma}_{a}=\frac{L \cos \phi}{m V_{a}}-\frac{g \cos \gamma_{a}}{V_{a}}+\sin ^{2} \gamma_{a} \cos \left(\psi-\psi_{d W}\right) \frac{\mathrm{d} W}{\mathrm{~d} z} \\
\dot{\psi}=\frac{L \sin \phi}{m V_{a} \cos \gamma_{a}}+\tan \gamma_{a} \sin \left(\psi-\psi_{d W}\right) \frac{\mathrm{d} W}{\mathrm{~d} z}
\end{gathered}
$$

Lift and drag are computed with the lift and drag coefficients $\left(C_{L}, C_{D}\right)$, through

$$
L=C_{L} \rho S V_{a}^{2} / 2, \quad D=C_{D} \rho S V_{a}^{2} / 2
$$

where $\rho$ is the air density and $S$ is the aircraft wing area. The aerodynamic coefficients are assumed to follow a parabolic drag polar (i.e., the drag coefficient is quadratic with respect to the lift coefficient),

$$
C_{D}=C_{D_{0}}+K C_{L}^{2}
$$

where $C_{D_{0}}$ is the parasitic drag coefficient and $K C_{L}^{2}$ is the induced drag coefficient. Note that

$$
K=\left(4\left(\frac{L}{D}\right)_{\max }^{2} C_{D_{0}}\right)^{-1}
$$

where $(L / D)_{\max }$ is the maximum lift over drag ratio, which, together with $C_{D_{0}}$, are the most important aircraft aerodynamic efficiency parameters and fully define the aerodynamic drag polar.

The aircraft energy state is defined by the sum of its potential and kinetic energy:

$$
E=m g h+m \frac{V_{a}^{2}}{2}
$$

\section{Conditions for Sustainable Flight}

Definition III.1 (sustainable flight): A finite duration flight trajectory is sustainable with respect to the safety altitude $h_{\min }$ and the minimum aircraft energy $E_{\min }$ if it satisfies Eqs. (ㅁ) and (ㅁ) and satisfies pointwise in time the following inequalities:

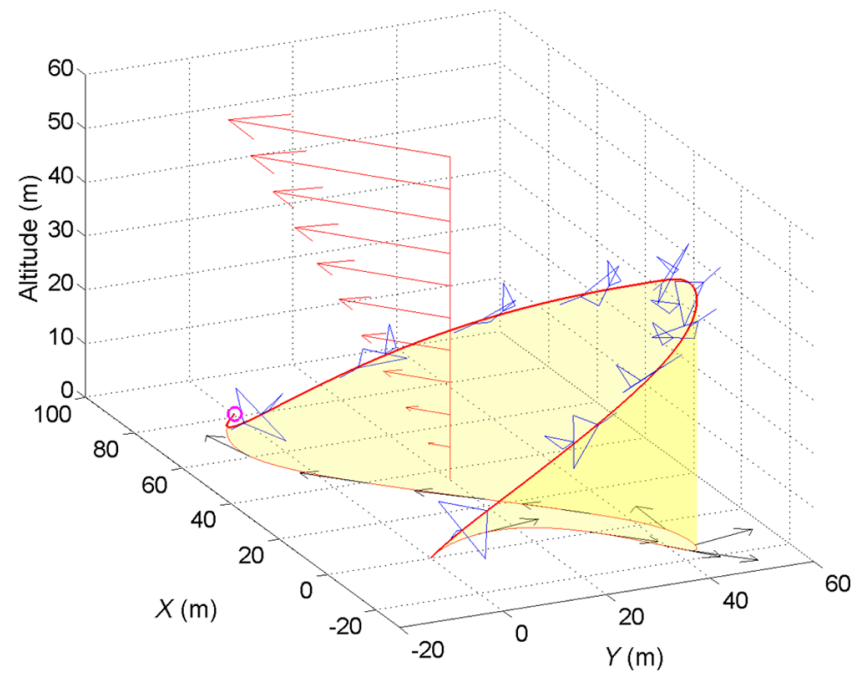

Fig. 4 Optimal dynamic soaring $S$ trajectory.

$$
\begin{aligned}
& h(t) \geq h_{\min }, \quad t \in\left[t_{i}, t_{f}\right] \\
& E(t) \geq E_{\min }, \quad t \in\left[t_{i}, t_{f}\right] \\
& E_{\min }:=m g h_{\min }+m \frac{V_{\min }^{2}}{2}
\end{aligned}
$$

where $t_{i}$ and $t_{f}$ are the initial and final times of the cycle, respectively, and $V_{\min }$ is the minimum velocity required to maintain sustained flight (i.e., the stall speed at $h_{\min }$ ).

Definition III.2 (flight cycle): A flight cycle is a trajectory of finite duration, satisfying Eqs. (4) and (6), where one or more of the aircraft state [Eq. (6)] and energy [Eq. (9)] variables have the same value at the initial and final times, and their initial and final time derivatives have the same sign. Hence, a cyclic variable $\kappa$ in a flight cycle satisfies

$$
\begin{gathered}
\kappa\left(t_{f}\right)=\kappa\left(t_{i}\right) \\
\operatorname{sgn}\left(\dot{\kappa}\left(t_{f}\right)\right)=\operatorname{sgn}\left(\dot{\kappa}\left(t_{i}\right)\right)
\end{gathered}
$$

where $t_{i}$ and $t_{f}$ are the initial and final times of the cycle, respectively.

Remark III.1: For wind shear soaring cycles, the cyclic variables are usually the course, bank, and pitch angles.

From here on, the execution of a sustainable flight through dynamic soaring is just referred to as dynamic soaring.

\section{A. Sufficient Conditions for Dynamic Soaring}

This work studies what the minimum vertical wind gradient $\mathrm{d} W / \mathrm{d} z$ (which enables an aircraft to perform dynamic soaring) is, if all other system parameters are fixed. It treats the most general dynamic soaring motion, three-dimensional dynamic soaring, where the trajectory is not constrained to any plane of motion and the flight is not constrained to be steady.

To define the necessary and sufficient conditions, the minimum wind gradient $(\mathrm{d} W / \mathrm{d} z)_{\min }$ is set as an explicit function of all the other parameters (independent variables). The computation of the minimum wind gradient for the domain of the independent variables is complex. Hence, a heuristic method was implemented that computes lower bounds in the conditions to perform dynamic soaring (i.e., sufficient conditions). To that end, solutions for cyclic flight trajectories need to be computed. For dynamic soaring, these trajectories present equal altitude and aircraft energy at the beginning and end. The method implemented to find such trajectories uses GPOPS 2 [5], a control optimization tool, with the linear wind gradient [Eq. (3)] and the aircraft model presented in Sec. II.B. 


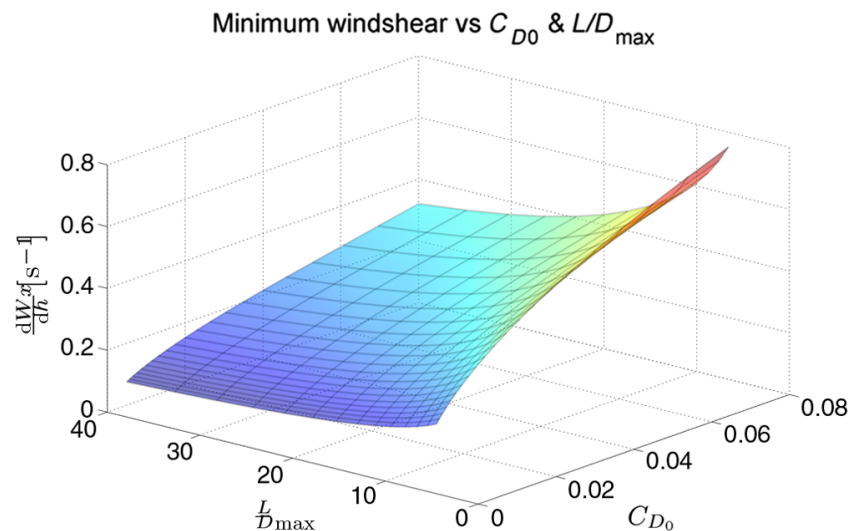

Fig. 5 Minimum wind gradient versus $(L / D)_{\max }$ and $C_{D_{0}}$.

The problem variables are separated into parameters that enter directly in dynamic equations and variables that define inequality constraints. To characterize the aerodynamic properties of the aircraft, and, in particular, the drag polar curve, maximum lift-to-drag ratio $(L / D)_{\max }$ and minimum drag coefficient $C_{D_{0}}$ were chosen. The other parameters are aircraft mass $m$, aircraft wing area $S$, air density $\rho$, and gravity acceleration $g$. The variables that define the inequality constraints are maneuvering limits, such as maximum lift coefficient, maximum wing loading, limit bank angle, and limit bank angular rate.

The characterization of the minimum vertical wind gradient dependence on environmental and aircraft parameters was obtained by running multiple trajectory optimizations, evaluating a range of values for several parameters. The analysis baseline parameters are $(L / D)_{\max }$ and $C_{D_{0}}$. The variables that define the inequality constraints are not as relevant, because they may be inactive. In fact, for analysis of the optimal trajectory, the inequality constraints were rendered inactive. The other parameters, like aircraft mass and gravity acceleration, do not need to be surveyed, because they are used to normalize the wind gradient through the nondimensional number

$$
\left(\frac{\mathrm{d} W}{\mathrm{~d} z}\right)^{2} \frac{2 m}{g \rho S}
$$

as presented in [2].

Hence, for each pair $\left[(L / D)_{\max }, C_{D_{0}}\right]$ in the parameters sweep, the optimization problem is defined by predetermined environmental and aerodynamic parameters, and the optimization conditions

Given:

$$
\left(\frac{L}{D}\right)_{\max }, \quad C_{D_{0}}
$$

Find:

$$
\left(\frac{\mathrm{d} W}{\mathrm{~d} z}\right)_{\min }=\min _{C_{L}(t), \phi(t), \psi_{d W}}\left\{\frac{\mathrm{d} W}{\mathrm{~d} z}: h(t) \geq h(0), \quad \forall t\right\}
$$

Figure 4 illustrates the optimal flight cycle, where the energy gained from the wind gradient matches exactly the energy lost due to drag. The optimal dynamic soaring trajectory presents an "S" shape. The aircraft starts at the lowest altitude turning toward the wind. It then climbs against the increasing wind. Near the top of the trajectory, the aircraft approaches stall speed, turns, and starts to descend with a decreasing wind. The aircraft then initiates a turn to restart the cycle.

Figure 5 illustrates the variation of the minimum vertical wind gradient with the aircraft $(L / D)_{\max }$ and $C_{D_{0}}$. The minimum wind gradient is illustrated here for an aircraft with the mass and wing area characteristics of a Cularis UAV $\$$ ( $\left.m=2.1 \mathrm{~kg}, S=0.55 \mathrm{~m}^{2}\right)$ and for the environmental conditions $g=9.8066 \mathrm{~m} / \mathrm{s}^{2}$ and $\rho=1.2041 \mathrm{~kg} / \mathrm{m}^{3}$. From

${ }^{\S}$ Data available online at http://lsts.fe.up.pt/vehicles/cularis [retrieved June 2013]. these parameters' sweep, it is possible to establish a lower bound on the conditions for dynamic soaring.

Theorem 1: The sufficient conditions for dynamic soaring in a linear wind shear are met if the aerodynamic, structural, and maneuvering inequality constraints are inactive and if

$$
\left(\frac{\mathrm{d} W}{\mathrm{~d} z}\right)^{2} \frac{2 m}{g \rho S} \geq k_{1} \tan \frac{k_{2} C_{D_{0}}}{(L / D)_{\max }}+k_{3}
$$

where $k_{1}=48.33, k_{2}=0.6793$, and $k_{3}=-2.66 \times 10^{-4}$.

\section{B. Necessary Conditions for Dynamic Soaring}

The necessary conditions for dynamic soaring can be defined by a similar heuristic to that used for the sufficient conditions. However, because a numerical optimization method is being used, one cannot prove analytically that the obtained solutions are in fact optimal and represent the necessary conditions. Nonetheless, it is possible to show that there is a very high level of confidence on this assertion, which is enough for results intended for engineering applications. To that end, one makes use of the law of large numbers and results from a Monte Carlo sampling of trajectories, environment parameters, and aircraft parameters to obtain a set of independent and identically distributed samples.

Consider the random variable

$$
X=\left\{\begin{array}{lc}
1, & \Delta h \geq 0 \\
0, & \text { otherwise }
\end{array}\right.
$$

where $\Delta h$ is the altitude difference between the beginning and end of a dynamic soaring flight cycle, and $\Delta h<0$ means that the flight cycle is not sustainable because the aircraft is losing altitude. Each random trial $\left(X_{i}\right)$ is a Bernoulli trial, with expected value and variance:

$$
\begin{gathered}
E[X]=\mu \\
\operatorname{var}(X)=\sigma^{2}=\mu(1-\mu)
\end{gathered}
$$

The claim that a certain condition is in fact a necessary condition is supported if $\mu \rightarrow 0$. Suppose that the Monte Carlo sampling shows that

$$
\begin{aligned}
& E\left[\frac{\sum^{N} X_{i}}{N}\right]=0 \\
& \operatorname{var}\left(\frac{\sum^{N} X_{i}}{N}\right)=0
\end{aligned}
$$

and in fact $\mu \rightarrow 0$ with any sample size $N$. The law of large numbers states that

$$
P\left(\left|\frac{\sum^{N} X_{i}}{N}-\mu\right| \geq \epsilon\right) \leq \frac{\sigma^{2}}{N \cdot \epsilon^{2}}=1-\beta
$$

where $\epsilon$ is the margin of error and $\beta$ is the confidence level. Consider the variance for the worst-case scenario, assuming that the $N+$ first trial would yield a $X_{N+1}=1$ :

$$
\sigma^{2} \leq \frac{1}{N}\left(1-\frac{1}{N}\right) \approx \frac{1}{N}
$$

As $\mu \rightarrow 0$,

$$
P\left(\left|\frac{\sum^{N} X_{i}}{N}\right| \geq \epsilon\right) \leq \frac{1}{N^{2} \cdot \epsilon^{2}}
$$

resulting in 


$$
\frac{1}{N^{2} \cdot \epsilon^{2}}=1-\beta \quad \Leftrightarrow N=\frac{1}{\epsilon \sqrt{(1-\beta)}}
$$

which means that, for a margin of error of 0.001 and a confidence level of $98.0 \%, N \geq 7072$ samples are needed. That means that, with a high degree of certainty, no more than one trial in a hundred yields $X_{N+1}=1$.

Hypothesis III.1: The necessary conditions for dynamic soaring in a linear wind shear are only met if the aerodynamic, structural, and maneuvering inequality constraints are inactive, and if

$$
\left(\frac{\mathrm{d} W}{\mathrm{~d} z}\right)^{2} \frac{2 m}{g \rho S} \geq k_{1} \tan \frac{k_{2} C_{D_{0}}}{(L / D)_{\max }}+k_{3}
$$

where $k_{1}=48.33, \quad k_{2}=0.6231, \quad$ and $\quad k_{3}=-2.70 \cdot 10^{-4}$, $(L / D)_{\max } \in[6.6,40]$, and $C_{D_{0}} \in[0.005,0.08]$.

These conditions were obtained by establishing lower bound conditions for the dynamic soaring. That was achieved by sweeping the aircraft parameters $(L / D)_{\max }$ and $C_{D_{0}}$ (Fig. 5), similar to the method used to define the sufficient conditions.

To check this hypothesis, a Monte Carlo simulation was run, which was set up as follows. For each run, a test dynamic soaring number DS is generated:

$$
\begin{gathered}
\mathrm{DS}_{i}=\mathrm{MC}_{i} \cdot\left(k_{1} \tan \frac{k_{2} C_{D_{0}}}{(L / D)_{\max }}+k_{3}\right), \\
\mathrm{MC}_{i} \sim \mathcal{U}(x): x \in[0,1)
\end{gathered}
$$

where $C_{D_{0}}$ and $(L / D)_{\max }$ are uniformly sampled from the intervals $[0.005,0.08]$ and $[6.6,40]$, respectively, and $\mathcal{U}(x)$ is the uniform distribution over the interval $x \in[0,1) . \mathrm{MC}_{i}$ is a random variable sampled from the uniform distribution on the interval $[0,1)$, because one wants to test for any condition under the necessary conditions. The dynamic soaring number is also

$$
\mathrm{DS}_{i}=\left(\frac{\mathrm{d} W}{\mathrm{~d} z}\right)^{2} \frac{2 m}{g \rho S}
$$

where $m, g, \rho$, and $S$ are sampled from uniform distributions:

$$
\begin{gathered}
m \sim \mathcal{U}([1,30]) \mathrm{kg} \\
g \sim \mathcal{U}([9,10]) \mathrm{m} / \mathrm{s}^{2} \\
\rho \sim \mathcal{U}([0.8,1.4]) \mathrm{kg} / \mathrm{m}^{3} \\
S \sim \mathcal{U}([0.5,2]) \mathrm{m}^{2}
\end{gathered}
$$

getting the test $\mathrm{d} W / \mathrm{d} z$ from Eq. (23).

The next step is to define a test trajectory. The initial trajectory hypotheses are sampled from a pool of figure S-shaped trajectories. Each initial trajectory is used in the GPOPS 2 optimizer [5] to obtain a most efficient trajectory for the parameters obtained from Eqs. (22) and (24). The higher the final flight cycle altitude, the more efficient a trajectory is. The initial and final altitudes are then compared to check the random variable $X$ [Eq. (14)], the desired sample.

As expected, the result for a Monte Carlo sampling with 8000 samples shows no sample equal to one, meaning that no sustainable flight cycle was found to contradict the necessary conditions [Eq. (24)].

Experimental Verified Conclusion 1: Hypothesis III.1 is verified with margin of error of $0.1 \%$ and a confidence level of $98.4 \%$.

\section{Conclusions}

The study is focused on sustainable aircraft trajectories through wind shear with a linear vertical wind gradient. The necessary and sufficient conditions for dynamic soaring are defined by the minimum wind gradient required. This study computes the minimum wind gradient required to execute sustainable dynamic soaring and analyzes how it depends on several aircraft and environmental parameters. The minimum wind gradient is proportional to wing area, air density, and gravity acceleration and is inversely proportional to aircraft mass. As expected, the minimum wind gradient decreases for more efficient aircraft (i.e., it grows with increasing aircraft parasitic drag coefficient $C_{D_{0}}$ and it decreases with increasing maximum liftto-drag ratio $(L / D)_{\max }$.

The sufficient conditions for dynamic soaring are computed through a heuristic method that optimizes the flight trajectories for different parameters (aircraft and environment). The necessary conditions are supported by verification with a Monte Carlo sampling method. The random sampling results in a high level of confidence on the necessary conditions.

\section{Acknowledgments}

The research leading to this work was funded in part by the U.S. Air Force grant FA 8650-07-2-3744. The authors gratefully acknowledge the support of the Aerospace and Robotic Controls Laboratory researchers at the University of Michigan. We further acknowledge the support of the AsasF group and the researchers from the Underwater Systems and Technology Laboratory at the University of Porto and the Portuguese Air Force Academy.

\section{References}

[1] Lawrance, N. R., and Sukkarieh, S., "Simultaneous Exploration and Exploitation of a Wind Field for a Small Gliding UAV," AIAA Paper 2010-8032, Aug. 2010.

[2] Zhao, Y. J., "Optimal Patterns of Glider Dynamic Soaring," Optimal Control Applications and Methods, Vol. 25, No. 2, 2004, pp. 67-89. doi:10.1002/(ISSN)1099-1514

[3] Sachs, G., "Minimum Shear Wind Strength Required for Dynamic Soaring of Albatrosses," Ibis - The International Journal of Avian Science, Vol. 147, No. 1, 2005, pp. 1-10.

[4] Sachs, G., and da Costa, O., "Dynamic Soaring in Altitude Region Below Jet Streams,” AIAA Paper 2006-6602, Aug. 2006.

[5] Patterson, M. A., and Rao, A. V., GPOPS - II Version 1.0: A GeneralPurpose MATLAB Toolbox for Solving Optimal Control Problems Using the Radau Pseudospectral Method, Univ. of Florida, Gainesville, FL, 2013. 